\title{
Investigation of the serum levels of anterior pituitary hormones in male children with autism
}

\author{
Keiko Iwata ${ }^{1 \dagger}$, Hideo Matsuzaki ${ }^{1{ }^{*}}$, Taishi Miyachi ${ }^{1}$, Chie Shimmura', Shiro Suda', Kenji J Tsuchiya', \\ Kaori Matsumoto ${ }^{1}$, Katsuaki Suzuki ${ }^{1}$, Yasuhide Iwata ${ }^{2}$, Kazuhiko Nakamura ${ }^{2}$, Masatsugu Tsujii ${ }^{1,3}$, Toshirou Sugiyama ${ }^{4}$ \\ , Kohji Sato ${ }^{5}$ and Norio Mori, ${ }^{1,2}$
}

\begin{abstract}
Background: The neurobiological basis of autism remains poorly understood. The diagnosis of autism is based solely on behavioural characteristics because there are currently no reliable biological markers. To test whether the anterior pituitary hormones and cortisol could be useful as biological markers for autism, we assessed the basal serum levels of these hormones in subjects with autism and normal controls.

Findings: Using a suspension array system, we determined the serum levels of six anterior pituitary hormones, including adrenocorticotropic hormone and growth hormone, in 32 drug-naive subjects (aged 6 to 18 years, all boys) with autism, and 34 healthy controls matched for age and gender. We also determined cortisol levels in these subjects by enzyme-linked immunosorbent assay. Serum levels of adrenocorticotropic hormone, growth hormone and cortisol were significantly higher in subjects with autism than in controls. In addition, there was a significantly positive correlation between cortisol and adrenocorticotropic hormone levels in autism.
\end{abstract}

Conclusion: Our results suggest that increased basal serum levels of adrenocorticotropic hormone accompanied by increased cortisol and growth hormone may be useful biological markers for autism.

\section{Introduction}

Autism is a neurodevelopmental disorder, categorised as a pervasive developmental disorder, and is characterised by severe and sustained impairment in social interaction, by deviance in communication, and patterns of behaviour and interest. The aetiology of autism is not well understood, although it is thought to involve genetic, immunologic and environmental factors [1]. The diagnosis of autism is based solely on behavioural characteristics, as there is currently no biological marker for autism.

Several studies have examined anterior pituitary hormones as possible biological markers for autism [2-8]. The anterior pituitary gland synthesises and secretes adrenocorticotropic hormone (ACTH), growth hormone (GH), follicle-stimulating hormone (FSH), luteinizing hormone (LH), thyroid-stimulating hormone (TSH) and prolactin (PRL). Of these hormones, ACTH deserves special

\footnotetext{
* Correspondence: matsu@hama-med.ac.jp

+ Contributed equally

'Research Center for Child Mental Development, Hamamatsu University

School of Medicine, Hamamatsu, Japan Full list of author information is available at the end of the article
}

attention, because it is the hormone involved in the hypothalamic-pituitary-adrenal (HPA) axis, which may be affected in autism [3,4,6,9-11]. The HPA axis is the basis for emotion and social interaction, through the synthesis and/or release of corticotropin-releasing hormone, ACTH and cortisol. All previous studies that have measured basal ACTH levels in autism have shown an increase in the serum/plasma levels of this hormone [3,4,6,10], except for one study that showed no difference [7]. Unlike the results for $\mathrm{ACTH}$, the results for serum cortisol levels in autism are inconsistent, with studies reporting either no difference between patients and controls [3,5-7] or a decrease in patients $[4,10]$. With regard to the basal serum/plasma levels of other anterior pituitary hormones in autism spectrum disorders (ASDs), the results are again contradictory: a decrease in patients [7] or no difference from controls [4] for GH; a decrease in patients [12,13] or no difference from controls [7] for FSH; and no difference from controls for TSH and PRL $[2,4,7]$.

The conflicting findings in the measurement of anterior pituitary hormones in ASDs probably arise because of differences in the subject population. For instance, many
C Biomed Central 
studies used samples from both male and female patients; however, a recent systemic serum proteome profiling study pointed out that male and female patients with Asperger's disorder had distinct biomarker fingerprints [7]. Moreover, the secretion of anterior pituitary hormones may be modified by antipsychotic and antiepileptic medications [14-16], which often had not been taken into consideration in previous studies.

In this study, we assessed the basal concentrations of anterior pituitary hormone and cortisol in serum from male, drug-naïve subjects with autism.

\section{Methods}

\section{Ethics approval}

This study was approved by the ethics committee of the Hamamatsu University School of Medicine. All participants and their guardians were given a complete description of the study, and provided written informed consent before enrolment.

\section{Subjects}

In total, 32 boys with autism (aged 6 to 18 years) and 34 healthy controls matched for agen and gender participated in this study. All the participants were Japanese, born and living in the Aichi, Gifu or Shizuoka prefectures of central Japan.

Based on interviews and available records, including those from hospitals, the diagnosis of autism were made based on the Diagnostic and Statistical Manual, Fourth Revision, Text Revision (DSM-IV-TR) criteria. The Autism Diagnostic Interview-Revised (ADI-R) was also conducted by two of the authors (KJT and KM), both of whom are experienced and reliable at diagnosing autism with the Japanese version of the ADI-R. We also used the Wechsler Intelligence Scale for Children, Third Edition, to evaluate the intelligence quotient. Comorbid psychiatric illnesses were excluded by means of the Structured Clinical Interview for DSM-IV (SCID). Participants were excluded from the study if they had any symptoms of inflammation, a diagnosis of fragile X syndrome, epileptic seizures, obsessive-compulsive disorder, affective disorder, or any additional psychiatric or neurological diagnosis. All the autistic subjects were drug-naive, and were not taking any dietary supplements.

Healthy control subjects were recruited locally by an advertisement. All control subjects underwent a comprehensive assessment of their medical history to eliminate individuals with any neurological or other medical disorders. The SCID was also conducted to scrutinise any personal or family history of past or present mental illness. None of the control subjects initially recruited fulfilled any of these exclusion criteria.

Fasting blood samples were collected by venipuncture from all participants between 11.00 and 12.30 hours, and the samples were kept at room temperature for 30 minutes. The samples were then separated by centrifugation, divided into aliquots of $200 \mu \mathrm{l}$, and stored at $-80^{\circ} \mathrm{C}$ until use. Serum levels of anterior pituitary hormones were assayed using a suspension array system (Bio-Plex; BioRad, Hercules, CA, USA), with a panel of pituitary antibodies (Milliplex MAP Human Pituitary Panel; Millipore, Billerica, MA, USA). This system allows simultaneous identification of pituitary hormones with antibodies chemically attached to fluorescently labelled microbeads. The beads were resuspended in assay buffer, and the reaction mixture was quantified using a protein array reader (Bio-Plex; Bio-Rad). Serum levels of cortisol were determined using a commercially available sandwich ELISA kit (R\&D Systems, Inc., Minneapolis, MN, USA) according to the manufacturer's instructions.

\section{Statistical analysis}

Clinical characteristics (age, weight, height and body mass index (BMI)) were analysed using an unpaired $t$ test, after confirmation that there were no significant differences in variance as assessed by the F-test. Comparisons of concentrations of anterior pituitary hormones and cortisol between subjects with autism and controls were made using the Mann-Whitney $U$-test. In these multiple comparisons, a Bonferroni-adjusted nominal $P$-value threshold of 0.007 was used. Evaluation of the relationships between serum hormone levels and clinical variables or symptom profiles, and those between hormone levels, was performed using Spearman's rank correlation coefficient. Additionally, linear regression analyses were conducted to examine whether any change in the hormone levels could be accounted for by another variable, such as age or the levels of other hormones. Values of $P<0.05$ were considered significant. All statistical analyses were performed using SPSS software (version 12.0 J; IBM, Tokyo, Japan).

\section{Results}

The characteristics of all the participants are summarised in Table 1 . There were no significant differences in the distributions of age, weight, height or BMI between the autism group and the control group.

The serum levels of ACTH were $11.6 \pm 5.1 \mathrm{pg} / \mathrm{mL}$ in subjects with autism and $7.2 \pm 3.1 \mathrm{pg} / \mathrm{mL}$ in controls. Therefore, the level of ACTH in subjects with autism was significantly higher than that in controls $(U=185.0$, $P<0.001$, by Mann-Whitney $U$-test) (Table 1 , Figure 1A). The serum levels of $\mathrm{GH}$ in subjects with autism $(6495.4 \pm 9072.2 \mathrm{pg} / \mathrm{mL})$ were also significantly higher than those in controls $(1590.1 \pm 2447.5 \mathrm{pg} / \mathrm{mL} ; U=$ 305.0, $P=0.002$, Mann-Whitney $U$-test) (Table 1 , Figure 1B). We carried out regression analyses to test the effect of age and other hormones on GH levels, because these 


\begin{tabular}{|c|c|c|c|}
\hline & Control group $(n=34)$ & Autism group $(n=32)$ & $P$-value \\
\hline Age, years & $12.4 \pm 2.6(6$ to 18$)$ & $12.3 \pm 3.2(6$ to 18$)$ & NS \\
\hline Weight, kg & $42.3 \pm 14.3(15.6$ to 89.3$)$ & $41.8 \pm 15.0(17.5$ to 96.6$)$ & NS \\
\hline Height, cm & $150.8 \pm 14.8(111$ to 174$)$ & $148.7 \pm 17.9(110$ to 178$)$ & NS \\
\hline $\mathrm{BMI}, \mathrm{kg} / \mathrm{m}^{2}$ & $18.1 \pm 3.5(12.7$ to 32.4$)$ & $18.3 \pm 3.1(13.9$ to 30.5$)$ & NS \\
\hline \multicolumn{4}{|l|}{$\mathrm{ADI}-\mathrm{R}$} \\
\hline Domain A score & - & $20.2 \pm 4.9(10$ to 27$)$ & - \\
\hline Domain BV score & - & $13.6 \pm 3.9(8$ to 21$)$ & - \\
\hline Domain C score & - & $5.4 \pm 2.0$ (3 to 9$)$ & - \\
\hline Domain D score & - & $3.1 \pm 1.0(2$ to 5$)$ & - \\
\hline \multicolumn{4}{|l|}{ WISC-III } \\
\hline Verbal IQ & - & $91.3 \pm 21.6$ (48 to 133 ) & - \\
\hline Performance IQ & - & $95.3 \pm 21.1$ (47 to 131$)$ & - \\
\hline Full-scale IQ & - & $91.0 \pm 23.2(44$ to 134$)$ & - \\
\hline \multicolumn{4}{|c|}{ Anterior pituitary hormones } \\
\hline $\mathrm{ACTH}, \mathrm{pg} / \mathrm{mL}$ & $7.2 \pm 3.1$ (3.7 to 14.2$)$ & $11.6 \pm 5.1$ (3.7 to 26.3 ) & $<0.001$ \\
\hline $\mathrm{GH}, \mathrm{pg} / \mathrm{mL}$ & $1590.1 \pm 2447.5$ (34.8 to 13708.0$)$ & $6495.4 \pm 9072.2(30.7$ to 34811.5$)$ & 0.002 \\
\hline $\mathrm{FSH}, \mathrm{mIU} / \mathrm{mL}$ & $3.8 \pm 2.0(0.8$ to 8.1$)$ & $5.7 \pm 3.7(0.6$ to 16.4$)$ & NS \\
\hline $\mathrm{LH}, \mathrm{mIU} / \mathrm{mL}$ & $1.4 \pm 1.7(0.1$ to 7.1$)$ & $2.5 \pm 2.5$ (0.1 to 11.8$)$ & NS \\
\hline $\mathrm{TSH}, \mu \mathrm{IU} / \mathrm{mL}$ & $3.6 \pm 1.4(1.0$ to 7.4$)$ & $3.3 \pm 2.2$ (0.3 to 11.2 ) & NS \\
\hline $\mathrm{PRL}, \mathrm{ng} / \mathrm{mL}$ & $20.9 \pm 9.1$ (4.4 to 38.0 ) & $25.2 \pm 14.0(8.2$ to 66.9$)$ & NS \\
\hline Cortisol, ng/mL & $58.3 \pm 25.3(16.8$ to 116.8$)$ & $74.2 \pm 20.0(23.5$ to 101.5$)$ & 0.004 \\
\hline
\end{tabular}

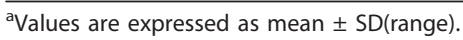

Abbreviations: BMI, body mass index; ADI-R, Autism Diagnostic Interview-Revised; WISC-III, the third edition of the Wechsler Intelligence Scale for Children; IQ, intelligence quotient; ACTH, adrenocorticotropic hormone; GH, growth hormone; FSH, follicle-stimulating hormone; LH, luteinizing hormone; TSH, thyroidstimulating hormone; PRL, prolactin; NS, not significant.

may affect GH levels [17-19]. After controlling for age and measured hormone levels (FSH, LH, TSH, PRL, $\mathrm{ACTH}$ and cortisol), we confirmed a significant difference in GH levels $\left(F_{(1,63)}=9.504, P=0.003\right.$ for age; $F_{(1,63)}=7.238, P=0.009$ for FSH; $F_{(1,63)}=8.429, P=$ 0.005 for LH; $F_{(1,63)}=9.891, P=0.003$ for TSH; $F_{(1,63)}=$ 9.033, $P=0.004$ for PRL; $F_{(1,62)}=6.611, P=0.013$ for $\mathrm{ACTH}$; and $F_{(1,60)}=4.687, P=0.034$ for cortisol) between subjects with autism and controls. There were no significant differences in FSH, LH, TSH or PRL levels between autistic and control subjects (Table 1).

The serum levels of cortisol were $74.2 \pm 20.0 \mathrm{ng} / \mathrm{mL}$ in subjects with autism and $58.3 \pm 25.3 \mathrm{ng} / \mathrm{mL}$ in controls. Therefore, the level of cortisol in subjects with autism was significantly higher than that in controls $(U=289.0$, $P=0.004$, Mann-Whitney $U$-test) (Table 1, Figure $1 C$ ). There was a significantly positive correlation between cortisol and ACTH levels in subjects with autism $\left(r_{s}=\right.$ $0.562, P<0.001$, Spearman's rank correlation coefficient) (Figure 1D). We also examined the correlations between serum ACTH, GH and cortisol levels and the symptom profiles in subjects with autism. The ADI-R domain A, $\mathrm{BV}$, and $\mathrm{C}$ scores were used as the symptom profiles. There were no significant correlations between the levels of any of the hormones and the symptom profiles (data not shown).

\section{Discussion}

We found that serum levels of ACTH and cortisol in subjects with autism were significantly higher than those in healthy controls. When the relationship between the levels of ACTH and cortisol was examined in subjects with autism, the levels of ACTH were significantly and positively correlated with the levels of cortisol, suggesting that in autism, cortisol secretion may be upregulated by increasing ACTH through the HPA axis [20]. It is possible that people with autism respond to the stress of venipuncture with activation of the HPA axis, leading to the elevation of ACTH; however, in this study, we found that the venipuncture effect was the same in autistic and control subjects, and therefore the observed differences are more likely to be due to the pathology of autism than to acute stress.

An increase in ACTH levels in people with autism is the most consistent result reported from studies of anterior pituitary hormones $[3,4,6,10]$. In functional imaging studies of the limbic system, which is the neural basis of emotions and social interactions, people with autism have been shown to have impaired circuitry in extinguishing fear responses [21]. Because the limbic system influences the HPA axis [22], the abnormal levels of $\mathrm{ACTH}$ and cortisol may be due to alterations in limbic system function $[8,23]$. 


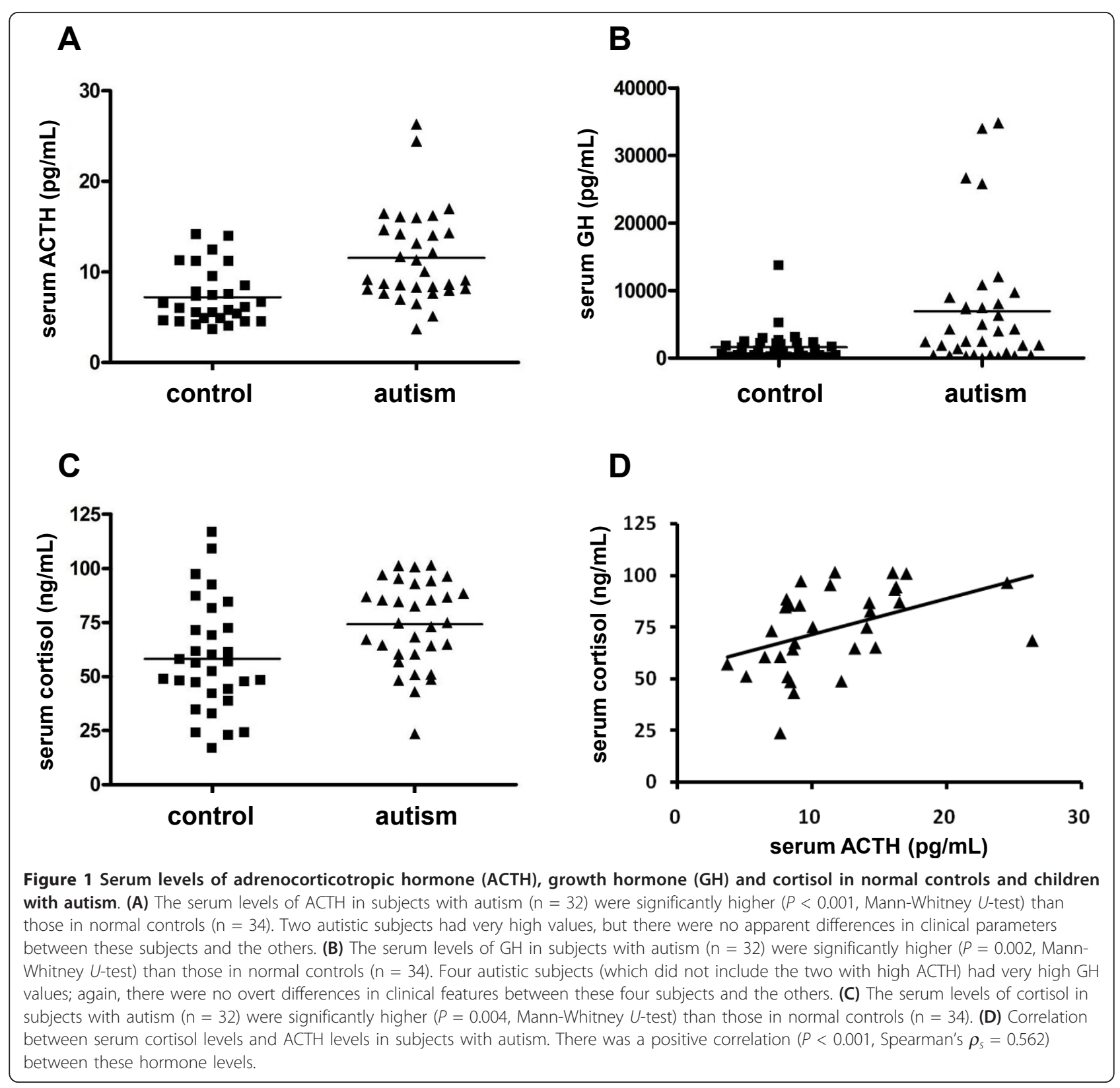

Unlike the present study, in which we found high levels of cortisol in subjects with autism, previous studies have reported low or no overt change in cortisol levels in autism $[3-7,10]$. Cortisol levels can be modified by psychotropic medications $[14,15]$; we recruited drug-naive subjects in this study, and all the previous studies $[3,5,6]$, except one [4], have used drug-free subjects to examine cortisol levels. Therefore, it is unlikely that the discrepancy between the present and previous results arose because of differences in the medication status of the participants. This, in turn, suggests that an alternative explanation is required. The present study included only male subjects, whereas previous studies comprised both male and female subjects [3-6,10]. In addition, the age range of the participants of the present study (6 to 18 years) was different from that of some of the previous studies, which enrolled adults only [5-7]. Furthermore, we collected the blood samples at around midday, whereas previous studies used samples collected in the morning [3-6,10]. Gender [7], age [24] and sampling time [24] are all known to be important factors influencing the cortisol level.

We also found that serum levels of GH in subjects with autism were significantly higher than those in healthy controls. There are no available data to interpret this increased GH in basal conditions in autism. However, 
because the serum levels of glutamate have been shown to be increased in adults with autism [25], and because intravenous administration of excitatory amino acids stimulates GH secretion [26-28], the increased basal GH levels in autism seen in our study may, at least in part, be due to a high concentration of glutamate in the circulation.

In this study, we found no significant correlations between cortisol levels and autistic symptoms as assessed by the ADI-R. This is in contrast to the results of Hamza et al. [10], who found an inverse correlation between hormone-stimulated plasma cortisol levels and the severity of autistic symptoms as assessed by the Childhood Autism Rating Scale. This discrepancy may be caused by the different scales used for the evaluation of clinical features.

There are some limitations to our study. The small sample size renders the data presented here preliminary. In addition, the study included only male participants. A larger study with subjects of both genders will be necessary, although separate analysis may still be warranted to eliminate the confounding effect of gender on hormone levels.

\section{Conclusion}

Our results suggest that increased basal serum levels of ACTH accompanied by increased cortisol and GH may be useful biological markers for autism.

\section{List of abbreviations}

ACTH: adrenocorticotropic hormone; ADI-R: Autism Diagnostic InterviewRevised; FSH: follicle-stimulating hormone; GH: growth hormone; HPA: hypothalamic-pituitary-adrenal axis; LH: luteinizing hormone; PRL: prolactin; SCID: Structured Clinical Interview for DSM-IV; TSH: thyroid-stimulating hormone.

\section{Acknowledgements}

This study was supported by a Grant-in-Aid for Scientific Research on Innovative Areas from the Ministry of Education, Culture, Sports, Science and Technology, Japan (to HM), a Grant-in-Aid for Scientific Research (B) from the Ministry of Education, Culture, Sports, Science and Technology of Japan (to NM) and a Grant-in-Aid for Young Scientists (B) from the Ministry of Education, Culture, Sports, Science and Technology of Japan (to KI).

\section{Author details}

${ }^{1}$ Research Center for Child Mental Development, Hamamatsu University School of Medicine, Hamamatsu, Japan. ${ }^{2}$ Department of Psychiatry and Neurology, Hamamatsu University School of Medicine, Hamamatsu, Japan. ${ }^{3}$ Faculty of Contemporary Sociology, Chukyo University, Toyota, Japan. ${ }^{4}$ Department of Child and Adolescent Psychiatry, Hamamatsu University School of Medicine, Hamamatsu, Japan. ${ }^{5}$ Department of Anatomy and Neuroscience, Hamamatsu University School of Medicine, Hamamatsu, Japan.

\section{Authors' contributions}

$\mathrm{HM}, \mathrm{KI}, \mathrm{KS}$ at and NM designed this study. KN, MT and TS were involved in the recruitment of participants. HM, TM and KN collected blood samples. KTJ and KM conducted clinical evaluations. KI, HM, CS, SS and YI measured and analysed serum levels of hormones from the anterior pituitary gland. $\mathrm{Kl}, \mathrm{HM}$, KSuz, KSat and NM participated in manuscript preparation. All authors read and approved the final manuscript.

\section{Competing interests}

The authors declare that they have no competing interests.

Received: 9 April 2011 Accepted: 19 October 2011

Published: 19 October 2011

\section{References}

1. Volkmar FR, Lord C, Bailey A, Schultz RT, Klin A: Autism and pervasive developmental disorders. J Child Psychol Psychiatry 2004, 45:135-170.

2. Cohen DJ, Young JG, Lowe TL, Harcherik D: Thyroid hormone in autistic children. J Autism Dev Disord 1980, 10:445-450.

3. Tordjman S, Anderson G, McBride P, Hertzig M, Snow M, Hall L, Thompson S, Ferrari P, Cohen D: Plasma beta-endorphin, adrenocorticotropin hormone, and cortisol in autism. J Child Psychol Psychiatry 1997, 38:705-715.

4. Curin JM, Terzić J, Petković ZB, Zekan L, Terzić IM, Susnjara IM: Lower cortisol and higher ACTH levels in individuals with autism. J Autism Dev Disord 2003, 33:443-448.

5. Strous RD, Golubchik P, Maayan R, Mozes T, Tuati-Werner D, Weizman A, Spivak B: Lowered DHEA-S plasma levels in adult individuals with autistic disorder. Eur Neuropsychopharmacol 2005, 15:305-309.

6. Tani P, Lindberg N, Matto V, Appelberg B, Nieminen-von Wendt T, von Wendt L, Porkka-Heiskanen T: Higher plasma ACTH levels in adults with Asperger syndrome. J Psychosom Res 2005, 58:533-536.

7. Schwarz E, Guest PC, Rahmoune H, Wang L, Levin Y, Ingudomnukul E, Ruta L, Kent L, Spain M, Baron-Cohen S, Bahn S: Sex-specific serum biomarker patterns in adults with Asperger's syndrome. Mol Psychiatry 2010.

8. Spratt EG, Nicholas JS, Brady KT, Carpenter LA, Hatcher CR, Meekins KA, Furlanetto RW, Charles JM: Enhanced cortisol response to stress in children in autism. J Autism Dev Disord 2011.

9. Corbett BA, Mendoza S, Wegelin JA, Carmean V, Levine S: Variable cortisol circadian rhythms in children with autism and anticipatory stress. $J$ Psychiatry Neurosci 2008, 33:227-234.

10. Hamza RT, Hewedi DH, Ismail MA: Basal and adrenocorticotropic hormone stimulated plasma cortisol levels among Egyptian autistic children: relation to disease severity. Ital J Pediatr 2010, 36:71.

11. Jansen LM, Gispen-de Wied CC, van der Gaag RJ, van Engeland H: Differentiation between autism and multiple complex developmental disorder in response to psychosocial stress. Neuropsychopharmacology 2003, 28:582-590.

12. Geier DA, Geier MR: A clinical and laboratory evaluation of methionine cycle-transsulfuration and androgen pathway markers in children with autistic disorders. Horm Res 2006, 66:182-188.

13. Geier DA, Geier MR: A prospective assessment of androgen levels in patients with autistic spectrum disorders: biochemical underpinnings and suggested therapies. Neuro Endocrinol Lett 2007, 28:565-573.

14. Leskiewicz M, Budziszewska B, Lason W: Endocrine effects of antiepileptic drugs. Przegl Lek 2008, 65:795-798.

15. Levy AD, Van de Kar LD: Endocrine and receptor pharmacology of serotonergic anxiolytics, antipsychotics and antidepressants. Life Sci 1992, 51:83-94.

16. Madhusoodanan S, Parida S, Jimenez C: Hyperprolactinemia associated with psychotropics-a review. Hum Psychopharmacol 2010, 25:281-297.

17. Holsboer F: Psychiatric implications of altered limbic-hypothalamicpituitary-adrenocortical activity. Eur Arch Psychiatry Neurol Sci 1989, 238:302-322.

18. Mauras N, Rogol AD, Haymond MW, Veldhuis JD: Sex steroids, growth hormone, insulin-like growth factor-1: neuroendocrine and metabolic regulation in puberty. Horm Res 1996, 45:74-80.

19. Zachmann M: Assessment of growth hormone secretion in children. Keio J Med 1990, 39:173-186.

20. Jacobson L: Hypothalamic-pituitary-adrenocortical axis regulation. Endocrinol Metab Clin North Am 2005, 34:271-292, vii.

21. Sweeten $T L$, Posey DJ, Shekhar A, McDougle CJ: The amygdala and related structures in the pathophysiology of autism. Pharmacol Biochem Behav 2002, 71:449-455.

22. Dallman MF, Akana SF, Strack AM, Scribner KS, Pecoraro N, La Fleur SE, Houshyar H, Gomez F: Chronic stress-induced effects of corticosterone on brain: direct and indirect. Ann N Y Acad Sci 2004, 1018:141-150. 
23. Corbett BA, Schupp CW, Levine S, Mendoza S: Comparing cortisol, stress, and sensory sensitivity in children with autism. Autism Res 2009, 2:39-49.

24. Hardy R, Cooper MS: Adrenal gland and bone. Arch Biochem Biophys 2010, 503:137-145.

25. Shinohe A, Hashimoto K, Nakamura K, Tsujii M, Iwata Y, Tsuchiya KJ, Sekine Y, Suda S, Suzuki K, Sugihara G, et al: Increased serum levels of glutamate in adult patients with autism. Prog Neuropsychopharmacol Biol Psychiatry 2006, 30:1472-1477.

26. Estienne MJ, Schillo KK, Green MA, Hileman SM, Boling JA: N-methyl-d, Iaspartate stimulates growth hormone but not luteinizing hormone secretion in the sheep. Life Sci 1989, 44:1527-1533.

27. Gay VL, Plant TM: N-methyl-D,L-aspartate elicits hypothalamic gonadotropin-releasing hormone release in prepubertal male rhesus monkeys (Macaca mulatta). Endocrinology 1987, 120:2289-2296.

28. Shahab M, Nusser KD, Griel LC, Deaver DR: Effect of a single intravenous injection of N-methyl-D,L-aspartic acid on secretion of luteinizing hormone and growth hormone in Holstein bull calves. J Neuroendocrinol 1993, 5:469-473.

doi:10.1186/2040-2392-2-16

Cite this article as: Iwata et al.: Investigation of the serum levels of anterior pituitary hormones in male children with autism. Molecular Autism 2011 2:16.

\section{Submit your next manuscript to BioMed Central} and take full advantage of:

- Convenient online submission

- Thorough peer review

- No space constraints or color figure charges

- Immediate publication on acceptance

- Inclusion in PubMed, CAS, Scopus and Google Scholar

- Research which is freely available for redistribution

Submit your manuscript at www.biomedcentral.com/submit
Biomed Central 